Pacific Journal of Mathematics

LIE'S FUNDAMENTAL THEOREMS FOR LOCAL

AD KARL STrambach 


\title{
LIE'S FUNDAMENTAL THEOREMS FOR LOCAL ANALYTICAL LOOPS
}

\author{
KaRL H. HofmanN AND KaRL Strambach
}

\begin{abstract}
A central piece of classical Lie theory is the fact that with each local Lie group, a Lie algebra is associated as tangent object at the origin, and that, conversely and more importantly, every Lie algebra determines a local Lie group whose tangent algebra it is. Up to equivalence of local groups, this correspondence is bijective.

Attempts at the development of a Lie theory for analytical loops have not been entirely satisfactory in this direction, since they relied more or less on certain associativity assumptions. Here we associate with an arbitrary local analytical loop a unique tangent algebra with a ternary multiplication in addition to the standard binary one, and we call this algebra an Akivis algebra. Our main objective is to show that, conversely, for every Akivis algebra there exist many inequivalent local analytical loops with the given Akivis algebra as tangent algebra. We shall give a good idea about the degree of non-uniqueness. It is curious to note that, on account of this non-uniqueness, the construction is more elementary than in the case of analytical groups.
\end{abstract}

The First and Third Fundamental Theorems of Sophus Lie assert the following statements (Cf. [18], IV, Kap. 15, pp. 365-404):

THE FIRST THEOREM. Every local analytical group determines on its tangent vector space at the identity the structure of a unique Lie algebra with the Lie bracket given by the formula

$$
[x, y]=\lim _{t \rightarrow 0} t^{-2}\left(\frac{t x \circ t y}{t y \circ t x}\right)
$$

where the local group operation $\circ$ is transported into the tangent space, and where $\mathrm{g} / \mathrm{h}$ is written for $\mathrm{g} \circ \mathrm{h}^{-1}$.

THE THIRD THEOREM. If $L$ is a finite dimensional real Lie algebra, then there exists a local analytical group whose tangent Lie algebra according to the First Theorem is isomorphic to L. Moreover, local analytical groups with this property are locally isomorphic. 
A reasonable proof of the Third Theorem proceeds along these lines (see e.g. [10], Chap. II, [26]): In the algebra of formal power series in two non-commuting variables $X$ and $Y$, there is a well-defined power series $X * Y$ defined by the equation $\exp (X * Y)=\exp X \exp Y$. If $X * Y=$ $\sum_{n=1} H_{n}(X, Y)$, where $H_{n}(X, Y)$ are the homogeneous polynomials of degree $n$, being defined as the linear combinations of the monomials of degree $n$ occurring in the series, then it turns out that $H_{n}(X, Y)$ is always contained in the Lie algebra $L[X, Y]$ generated by the variables $X$ and $Y$ in the Lie algebra of all polynomials relative to the commutator bracket $[p, q]=p q-q p$. In particular $H_{1}(X, Y)=X+Y, H_{2}(X, Y)=\frac{1}{2}[X, Y]$, $H_{3}(X, Y)=\frac{1}{12}([X,[X, Y]]+[Y,[Y, X]])$. One shows further, that Lie $[X, Y]$ is in fact the free Lie algebra generated by $X$ and $Y$ so that for every given Lie algebra $L$ the polynomials $H_{n}(x, y)$ are well defined for any pair of elements $x, y \in L$. If we now consider a finite dimensional real Lie algebra $L$ we can introduce a norm which is compatible with the topology and which satisfies $\|[x, y]\| \leq\|x\|\|y\|$. Then one shows that the Campbell-Hausdorff series converges absolutely on the open ball $B$ with radius $\frac{1}{2} \log 2$. Thus one obtains an analytical function $(x, y) \mapsto x * y$ : $B \times B \rightarrow L$ which defines on $B$ the desired structure of a local group.

A loop is a group except that one allows the multiplication to be non-associative. Specifically: A loop is a set $G$ with a distinguished element $e$ and three binary operations $(x, y) \mapsto x y, x / y, y \backslash x: G \times G \rightarrow$ $G$ such that $x e=e x=x$ and $y(y \backslash x)=(x / y) y=x$ for all $x, y \in G$. Topological and analytical loops are easily defined, and with the usual circumspection one defines local analytical loops (see $\S 3$ below or [2]). In this paper we prove Lie's fundamental theorems for local analytical loops.

Why bother? There are several reasons. Firstly, Lie loops have been considered in the literature at least since that paper by A. I. Malcev [20] which is considered the start of the theory. The area of Moufang Lie loops is in fact a highly developed theory for which most results of classical Lie group theory have been verified mostly through the work of Kuzmin and Kerdman [14, 15, 16, 17] and through Sagle [22, 23, 24]. In his extensive study of webs in differential geometry, Akivis and his school [1 through 7, 21] contributed substantially to the general foundations of Lie loop theory. However, Lie's Third fundamental theorem was never established (see [3]). Holmes and Sagle studied local power associative Lie loops and dealt with the question to which extent the Campbell-Hausdorff formalism could be brought to bear on the theory [13]. A similar discussion is to be found in a paper by Akivis [1].

However, apart from the applications to Lie loop theory we feel that a discussion of Lie's fundamental theorems in the absence of associativity 
sheds new light on the classical associative case. In fact, before we can deal with the non-associative case we first have to find the appropriate generalization of the concept of a Lie algebra. The way in this direction was shown by Akivis. In (local) Lie group theory, the Lie bracket $[x, y]$ introduced in (1) measures the degree of commutativity of the local operation. It is Lie's most remarkable insight that the bracket is determined by the degree two terms in the Taylor expansion of the product, and that is suffices as a basis for the entire local theory. Akivis [3] has observed that, in the absence of associativity, one must measure the degree of associativity by a ternary operation called associator bracket:

$$
\langle x, y, z\rangle=\lim _{t \rightarrow 0} t^{-3}\left(\frac{(t x \circ t y) \circ t z}{t x \circ(t y \circ t z)}\right)
$$

and that the two are linked by an identity which we shall call the Akivis identity (cf. [3], [7])

$$
\begin{aligned}
& \sum_{g \in S_{3}} \operatorname{sgn}(g)\left\langle x_{g(1)}, x_{g(2)}, x_{g(3)}\right\rangle \\
& \quad=\left[\left[x_{1}, x_{2}\right], x_{3}\right]+\left[\left[x_{2}, x_{3}\right], x_{1}\right]+\left[\left[x_{3}, x_{1}\right], x_{2}\right]
\end{aligned}
$$

One remarks immediately that in the case of an associative local multiplication the associator bracket vanishes and the Akivis identity reduces to the classical Jacobi identity.

We shall say that an algebra with a bilinear anticommutative multiplication $(x, y) \mapsto[x, y]$ and a trilinear ternary multiplication is an Akivis algebra if it satisfies the Akivis identity (A). (Akivis calls these algebras $W$-algebras [3].) This concept is quite natural: If one considers an algebra $E$ over a field $F$ with a binary bilinear multiplication $(x, y) \mapsto x y$ and defines two new operations by $[x, y]=x y-y x$ and $\langle x, y, z\rangle=(x y) z-$ $x(y z)$, then the resulting algebra $(E,[\cdot, \cdot],\langle\cdot, \cdot, \cdot\rangle)$ is an Akivis algebra.

Thus a problem is whether for any finite dimensional real Akivis algebra we find a local analytical loop whose associated tangent Akivis algebra is the given one. Our answer is affirmative and reads, perhaps a bit surprisingly, as follows:

THEOREM. Let $W$ be a completely normable vector space over the field of real or complex numbers and suppose that we are given continuous multilinear maps $[\cdot, \cdot]: W^{2} \rightarrow W$ and $\langle\cdot, \cdot, \cdot\rangle: W^{3} \rightarrow W$ making $W$ into an Akivis algebra. Then there are continuous trilinear maps $r, s: W^{3} \rightarrow W$ such 
that the multiplication

$$
(x, y) \mapsto x \circ y=x+y+\frac{1}{2}[x, y]+r(x, x, y)+s(x, y, y): W^{2} \rightarrow W
$$

defines near 0 the structure of a local analytical loop in such a way that the commutator and associator bracket defined for it according to formulae (1) and (2) is the given one (so that the associated Akivis algebra is indeed the given one).

The proof is relatively elementary insofar at it is practically carried out in the domain of multilinear algebra which never goes beyond degree 3. Not only is this proof more elementary than any of the proofs of Lie's Third Theorem, but it also lacks the uniqueness statement. Even among the polynomial multiplications of degree three there are many pairs of trilinear maps $(r, s)$ which satisfy the condition of the Theorem. In fact, the variety of different solutions to the problem in the realm of polynomial maps of degree three is in bijective correspondence to the space of all continuous symmetric trilinear maps $W^{3} \rightarrow W$, as we will show. More ambiguity comes in if we modify one of the multiplications exhibited in the Theorem by allowing for additional summands of higher order than the third. All such will always define the same Akivis tangent algebra. In particular, if we start from any of the familiar finite dimensional Lie algebras, there are numerous local analytical loops whose associated Akivis algebra is the given Lie algebra and there are several among them whose multiplication is polynomial of total degree not more than three. However, up to local isomorphism, there will be only one which is associative, namely, the one guaranteed by Lie's Theorem and which we can obtain from the Campbell-Hausdorff series. If we start with an arbitrary Akivis algebra, the Campbell-Hausdorff formula will not in general give a local loop whose associated Akivis algebra is the given one. This is, however, the case if the given Akivis algebra is alternative - which means that any pair of its elements is contained in a Lie subalgebra. Since the relation between the proof of Lie's Third Theorem and the CampbellHausdorff formalism is so important, we will also comment on the Campbell-Hausdorff formalism. In this context we discuss the property of alternativity. We emphasize, however, that we do not wish to deal primarily with the effect of weak associativity hypotheses; we are interested in the Fundamental Theorems in the absence of any associativity assumption. Under such general circumstances it is not even known whether local one parameter subloops exist (although a recent theorem by Hofmann and Lawson shows that in any local analytical loop one can, perhaps after 
making the domain of the multiplication smaller, when necessary, always find a unique smallest closed local multiplicatively closed connected subset having a given half-line in the tangent algebra as its tangent space [11]). Our methods therefore do not rely on the traditional local Lie theory. We proceed as follows: In the first section we provide some background from multilinear algebra. Since the action of the symmetric group on the arguments of multilinear maps is crucial for our proof, we need some information on the representation theory of symmetric groups. The second section gives the proof of the Fundamental Theorems, however, in a purely algebraic setting of formal loops. We also comment on the Campbell-Hausdorff formula in the context of power-associative algebras. Indeed, power-associativity is the very least assumption we have to require to be even able to speak about the exponential function. We indicate a purely algebraic proof of a result of Holmes and Sagle's, according to which one can express the homogeneous components of the Campbell-Hausdorff series, in the context of non-associative power-associative algebras, completely inside the Akivis algebra associated with the algebra [13]. One can show in this context that a power-associative local Bruck loop (i.e. a loop satisfying $x^{-1}(x y)=(y x) x^{-1}=y$ ) is alternative. We formulate some problems which appear to be of urgency in the general context of Akivis algebras and any theory which would parallel that of Lie algebras and Lie groups. In the third section we utilize the accumulated information to give a proof of the Fundamental Theorems; given standard analysis results this is no longer difficult, since all the work is done in the algebraic context of the first two sections.

Acknowledgment. We thank the referee for his careful scrutiny of the paper, which has prevented an error in a lemma and which has eliminated a number of typographical errors.

1. A Lemma on multilinear algebra. Symmetry and antisymmetry of multilinear maps are governed by the actions of the symmetric group on the arguments. Hence we consider actions of the group $S_{n}$ (on $n$ elements) on vector spaces. The case that actually interests us is that of $n=3$. We will take a groundfield of characteristic zero. (A characteristic relatively prime to $n$ ! would suffice!) We define $R$ to be the group algebra $F\left[S_{n}\right]$. In $R$ we define the antisymmetry element $a \in R$

$$
a=\frac{1}{n !} \sum\left\{\operatorname{sgn}(g) \cdot g: g \in S_{n}\right\} .
$$


This element is idempotent, and if $A_{n}$ is the subgroup of even permutations in $S_{n}$, then $a g=a$ for $g \in A_{n}$ and $a g=-a$ for $g \notin S_{n} \backslash A_{n}$. We are interested in the right ideal $(1-a) R$ and note

(ii) $(1-a) R=\{x \in R: a x=0\}=$ annihilator of $a$ on the right.

In particular,

$$
\begin{gathered}
1+\left(S_{n} \backslash A_{n}\right) \subset(1-a) R, \\
1-A_{n} \subset(1-a) R .
\end{gathered}
$$

As the $n ! / 2+(n ! / 2-1)$ elements of $\left(1+\left(S_{n} \backslash A_{n}\right)\right) \cup\left(1-\left(A_{n} \backslash\{1\}\right)\right)$ are linearly independent, and since $a$ does not annihilate $a$ because of $a^{2}=a \neq 1$, we know

$$
\operatorname{dim}(1-a) R=n !-1 \text {. }
$$

If $H$ is a subgroup of $S_{n}$ and we set $e=(1 /|H|) \sum H$, then $e R$ is the fixed point set of $H$ on $R$ for left multiplication. If $T \subset S_{n}$ is a set which meets each coset $H g$ in precisely one point, then $S_{n}=H T$ and $e H s=\{e s\}$ for each $s \in T$, and thus $e R$ is spanned precisely by the [G:H] linearly independent elements es, $s \in T$. In particular, if $t$ is an involution $\left(t^{2}=1\right.$ $\neq t$ ), then this applies to $H=\{1, t\}$ and shows

$$
\operatorname{dim}(1+t) R=\operatorname{dim} \frac{1}{2}(1+t) R=\frac{n !}{2} .
$$

We are now ready for the following Lemma:

1.1. Lemma. Let $t$ denote a transposition of two elements in $S_{3}$ and $c \neq 1$ a cyclic permutation of all elements (i.e., and element of order 3). Then

$$
(1+t) R+(1+t c) R=(1-a) R \text {. }
$$

Proof. Since $n=3$, we know that $t c$ is again an involution. Hence the left hand side of (1) is contained in the right hand side by (iii). By (iv) it now suffices to show that the dimension of the left hand side is $n !-1$. Since $\operatorname{dim}(1+t) R=\operatorname{dim}(1+c t) R=n ! / 2$ by (v) it suffices to show that $(1+t) R \cap(1+c t) R$ is one dimensional. But $(1+t) R$ is the precise fixed point set of $t$ and $(1+c t) R$ is the precise fixed point set of $c t$ (under multiplication on the left). Hence the intersection of the two set is the fixed point set of the subgroup generated by $t$ and $t c$, which is all of $S_{n}$. But this fixed point set is precisely $e R$ with $e=(1 / n !) \sum S_{n}$, and hence is one dimensional. 
We observe that Lemma 1.1 generalizes to more general groups $G$ in place of $S_{3}$ : Indeed if $G$ is dihedral, i.e., contains a cyclic normal subgroup $C=\langle c\rangle$ of order $m \geq 3$ and of index 2 such that $G / C$ acts on $C$ by inversion, then we let sgn: $G \rightarrow\{1,-\}$ denote the homomorphism with kernel $C$ and note that every element $t \in G \backslash C$ is an involution. If we replace $n$ ! by $|G|=2 m$, then the Lemma remains intact.

The identity (1) in Lemma 1.1 is of the form $I+J=e R$ for two right ideals $I$ and $J$ and an idempotent $e$ in a ring $R$. The following Lemma generalizes it to the corresponding relation $I V+J V=e V$ for any arbitrary left $R$-module $V$. Just as Lemma 1.1, we formulate it, however, in the more narrow frame which we need in Corollary 1.5 below, and this in turn is all that is used later in the paper.

1.2. LemMA. Let $V$ be an arbitrary left $S_{3}$-module over $F$, hence an $R=F\left[S_{3}\right]$-left-module. Then

$$
(1+t) V+(1+t c) V=(1-a) V
$$

for any element $t, c \in S_{3}$ of order 2 and 3 , respectively.

Proof. We want to reduce the proof to the information gathered in Lemma 1 . We recall that the left $R$-module $V$ is isomorphic to the left module $R \otimes_{R} V$ under the map $v \mapsto 1 \otimes v: V \rightarrow R \otimes_{R} V$ (with the inverse map $x \otimes v \mapsto x v$ ), where the $R$-left-multiplication on $R \otimes_{R} V$ is given by $x(y \otimes v)=x y \otimes v$, and where, in the formation of the tensor product $R$ is considered as a $R$-right-module. Since every submodule of an $R$-module splits by Maschke's theorem, the functor $-\otimes_{R} V$ from the category of $R$-right-modules to the category of $F$-vector spaces is exact. We now consider the sequence of $R$-right-modules.

(3) $R \oplus R \stackrel{f}{\rightarrow} R \stackrel{f^{\prime}}{\rightarrow} R, \quad f(x, y)=(1+t) x-(1+c t) y, \quad f^{\prime}(x)=a x$.

To say that (3) is exact is precisely the statement of Lemma 1.1. Hence upon applying $-\otimes_{R} V$ to (3) we obtain the exact sequence of $F$-vector spaces

$$
(R \oplus R) \otimes_{R} V \stackrel{f \times 1}{\rightarrow} R \otimes_{R} V \stackrel{f^{\prime} \times 1}{\rightarrow} R \otimes_{R} V .
$$

The function

$$
\begin{aligned}
h:(R \oplus R) \otimes_{R} V \rightarrow\left(R \otimes_{R} V\right)+ & \left(R \otimes_{R} V\right), \\
& h((x, y) \otimes v)=(x \otimes v, y \otimes v)
\end{aligned}
$$


induces an isomorphism of $R$-left-modules (see e.g. MacLane [19], p. 189, (1.6)ff).

Now we consider the following diagram:

$\begin{array}{llclc}V \oplus V & \stackrel{F}{\rightarrow} & V & \stackrel{F^{\prime}}{\rightarrow} & V \\ i \oplus i \uparrow & & i \uparrow & & i \uparrow\end{array}$

(5) $\quad\left(R \otimes_{R} V\right) \oplus\left(R \otimes_{R} V\right) \stackrel{F^{*}}{\rightarrow} \quad R \otimes_{R} V \stackrel{f^{\prime} \otimes 1}{\rightarrow} \quad R \otimes_{R} V$

$\begin{array}{cccc}h \uparrow & 1 \uparrow & 1 \uparrow \\ (R \oplus R) \otimes_{R} V & \stackrel{f \otimes 1}{\rightarrow} & R \otimes_{R} V \underset{f^{\prime} \otimes 1}{\rightarrow} & R \otimes_{R} V\end{array}$

with $F(v, w)=(1+t) v-(1+t c) w, F^{*}(p, q)=(1+t) p-(1+t c) q$, $F^{\prime}(v)=a v$, and $i(x \otimes v)=x v$.

Commutativity of this diagram is readily checked and all vertical maps are isomorphisms. Since the bottom line is the exact sequence (4), the top line sequence is exact. But this is just another expression for the assertion (2).

We remark that the maps $f \otimes 1, F$ and $F^{*}$ are only $F$-linear, but not $R$-linear.

1.3. REMARK. Under the circumstances of 1.2 and its proof, the kernel $\operatorname{ker} F$ of the map $F=((v, w) \mapsto(1+t) v-(1+t c) w)$ is isomorphic to $(\operatorname{ker} f) \otimes_{R} V$.

Proof. From the commutative diagram (5) we draw the information $\operatorname{ker} F=\operatorname{ker}(f \otimes 1)$. Since $-\otimes_{R} V$ is exact, we conclude $\operatorname{ker}(f \otimes 1)=$ $(\operatorname{ker} f) \otimes_{R} V$.

We have $\operatorname{dim} \operatorname{ker} f=\operatorname{dim} \operatorname{dom} f-\operatorname{dim} \operatorname{im} f=2 n !-(n !-1)=$ $n !+1(=7$, if $n=3)$, since $\operatorname{dom} f=R \oplus R$ and $\operatorname{im} f=(1+t) R+$ $(1+t c) R$.

We remark that $\operatorname{ker} F$ as well as $(\operatorname{ker} f) \otimes_{R} V$ are $F$-vector spaces, but not necessarily $R$-left-modules.

Now we consider arbitrary $F$-vector spaces $W_{1}$ and $W_{2}$ and define $V$ to be the $F$-vector space $\operatorname{Hom}\left(W_{1}^{n} ; W_{2}\right)$ of all multilinear maps 
$v: W_{1} \times \cdots \times W_{1} \rightarrow W_{2}$. Then $S_{n}$ acts on $V$ on the left by permutation of the arguments as follows:

(6) For $x \in S_{n}$ and $v \in V$ let $x v\left(w_{1}, \ldots, w_{n}\right)=v\left(w_{\bar{x}(1)}, \ldots, w_{\bar{x}(n)}\right)$, $\bar{x}=x^{-1}$. (If we write $w_{k}^{\prime}=w_{\bar{x}(k)}$, then

$$
\begin{aligned}
y(x v)\left(w_{1}, \ldots, w_{n}\right) & =y v\left(w_{1}^{\prime}, \ldots, w_{n}^{\prime}\right)=v\left(w_{\bar{y}(1)}^{\prime}, \ldots, w_{\bar{y}(n)}^{\prime}\right) \\
& =v\left(w_{\bar{x} \bar{y}(1)}, \ldots, w_{\bar{x} \bar{y}(n)}\right)=v\left(w_{\overline{y x}(1)}, \ldots, w_{\overline{y x}(n)}\right) \\
& \left.=(y x)\left(w_{1}, \ldots, w_{n}\right) .\right)
\end{aligned}
$$

If we set $m=(1 / n !) \sum S_{n}$ and $e=(2 / n !) \sum A_{n}$, we notice $a m=m a=0$, $m+a=e$. A multilinear map $v$ is called symmetric iff $g v=v$ for all $g \in S_{n}$, equivalently $m v=v$. It is called antisymmetric iff $t v=-v$ for all transpositions $t$; this means $g v=\operatorname{sgn}(g) v$ for all $g \in S_{n}$, equivalently $a v=v$.

For each multilinear map $v$ we have $v=a v+(1-a) v=m v+$ $(1-m) v$; thus it can be represented as a sum of its antisymmetric part av and a complement $(1-a) v$ which is annihilated by $a$, and it can be written as a sum of its symmetric part $m v$ and a complement $(1-m) v$ which is annihilated by $m$. The symmetric parts of $v$ and $(1-a) v$ agree because of $a m=0$; likewise the antisymmetric parts of $v$ and $(1-m) v$ agree.

From our preceding Lemmas we now readily derive the following result:

1.4. Proposition. If $V$ denotes the F-vector space $\operatorname{Hom}\left(W_{1}^{3} ; W_{2}\right)$ of all 3-linear maps $v: W_{1}^{3} \rightarrow W_{2}$, then the space $(1-a) V$ of all 3-linear maps with zero antisymmetric part is $(1+t) V+(1+t c) V$ for each transposition $t$ and each 3-cycle $c$ in $S_{3}$.

This follows immediately from Lemma 1.2.

We now formulate 1.4 in the form we shall need it later:

1.5. Corollary. Every trilinear map $v: W_{1}^{3} \rightarrow W_{2}$ with zero antisymmetric part can be written in the form $v(x, y, z)=r(x, y, z)+r(y, x, z)-$ $s(x, y, z)-s(x, z, y)$ with two trilinear maps $r, s: W_{1}^{3} \rightarrow W_{2}$.

Proof. In 1.4 we take for $t$ the transposition $t=\left(\begin{array}{ll}1 & 2\end{array}\right)$, and for $c$ the cyclic permutation $\left(\begin{array}{lll}1 & 2 & 3\end{array}\right)$. Then $t c=\left(\begin{array}{ll}2 & 3\end{array}\right)$, and the assertion is a direct consequence of 1.4. 
In this very context we now consider the $F$-vector space $U=$ $\operatorname{Pol}^{3}\left(W_{1}, W_{2}\right)$ all homogeneous polynomial functions $p: W_{1} \times W_{1} \rightarrow W_{2}$ of degree 3 with the additional property that $p(x, 0)=p(0, x)=0$ for all $x$. This means that each $p \in U$ is of the form $p(x, y)=r(x, x, y)+$ $s(x, y, y)$ with a pair $(r, s) \in V \times V$. We define the linear function $G$ : $V \times V \rightarrow U$ by $G(r, s)(x, y)=r(x, x, y)+s(x, y, y)$ and notice that $(r, s) \in \operatorname{ker} G$ iff $r(x, x, y)+s(x, y, y)=0$ for all $x, y$; this condition implies $r(x+y, x+y, z)+s(x+y, z, z)=0$ for all $x, y, z$. A quick calculation shows that this implies $r(x, y, z)+r(y, x, z)=0$ for all $x, y, z$, and this is equivalent to $r(x, x, y)=0$ for all $x, y$. Similarly we obtain $s(x, y, y)=0$ for all $x, y$. Clearly, these conditions in turn imply

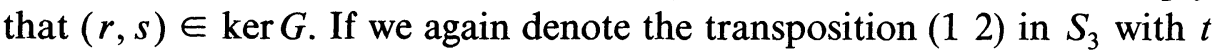
and the transposition (2 3) with $t c$, we have shown that

$$
\operatorname{ker} G=\operatorname{ker}(1+t) \oplus \operatorname{ker}(1+t c)
$$

where we use the expression $1+t$ for the $V$-endomorphism $v \mapsto(1+t) v$, and similarly for $1+t c$. In particular, we have $\operatorname{ker} G \subseteq \operatorname{ker} F$ with $F(v, w)$ $=(1+t) v-(1+t c) w$ as in 1.2 and 1.3. Now $G$ induces an isomorphism $G^{*}: V / \operatorname{ker}(1+t) \oplus V / \operatorname{ker}(1+t c) \rightarrow U$, and $F$ induces a morphism $F^{*}: V / \operatorname{ker}(1+t) \oplus V / \operatorname{ker}(1+t c) \rightarrow(1-a) V$. This allows us to define the morphism $\phi=F^{*} G^{*-1}: U \rightarrow(1-a) V$ which we know to be surjective after 1.4. and 1.5. The kernel of $F^{*} G^{*-1}$ is isomorphic to $\operatorname{ker} F^{*}$. We may replace $V / \operatorname{ker}(1+t)$ by its isomorphic copy $(1+t) V$, and $V / \operatorname{ker}(1+t c)$ by its isomorphic copy $(1+t c) V$; under this replacement, we have to replace $F^{*}$ by the map $D:(1+t) V \oplus(1+t c) V \rightarrow$ $(1-a) V, D(v, w)=v-w$. But the kernel of this morphism is isomorphic to $(1+t) V \cap(1+t c) V$ under the map $v \mapsto(v, v)$. We have observed earlier that $(1+t) V \cap(1+t c) V$ is the fixed point space of $t$ and of $t c$, which generate $S_{3}$. Hence it is the fixed point space $m V$ of all symmetric trilinear maps. We have shown:

1.6. Proposition. Let $V$ be the vector space of all trilinear maps $W_{1}^{3} \rightarrow W_{2}$, and consider the following vector subspaces:

(a) the subspace $a V$ of all antisymmetric maps,

(b) the subspace $(1-a) V$ of all trilinear maps without antisymmetric parts, and

(c) the subspace $\mathrm{mV}$ of all symmetric trilinear maps.

If further $U$ denotes the $F$-vector space of all homogeneous polynomial maps $p: W_{1}^{2} \rightarrow W_{2}$ of degree 3 with $p(0, x)=p(x, 0)$, then there are exact 
sequences

$$
\begin{aligned}
& 0 \rightarrow m V \rightarrow U \stackrel{\phi}{\rightarrow}(1-a) V \rightarrow 0, \\
& 0 \rightarrow m V \rightarrow U \stackrel{\phi}{\rightarrow} V \stackrel{a}{\rightarrow} a V \rightarrow 0 .
\end{aligned}
$$

If $W_{1}$ and $W_{2}$ are finite dimensional $F$-vector spaces of dimension $j$ and $k$, respectively, then elementary calculations show that $m V$ is the space of homogeneous polynomials of degree 3 in $j$ commuting variables, tensored with $W_{2}$; thus $m V$ is $\left(j^{2}+\left(\begin{array}{l}j \\ 3\end{array}\right)\right) k$-dimensional, while $a V$ may be identified with $\operatorname{Hom}\left(W_{1} \wedge W_{1} \wedge W_{1}, W_{2}\right)$, hence is $\left(\frac{j}{3}\right)$-dimensional. From the fact $(1-a) V$ is the kernel of $a: V \rightarrow a V$, it then follows that

$$
\operatorname{dim}_{F}(1-a) V=\operatorname{dim} V-\operatorname{dim} a V=j^{3} k-\left(\begin{array}{l}
j \\
3
\end{array}\right) k \text {. }
$$

Thus

$$
\begin{aligned}
\operatorname{dim} U & =\operatorname{dim} m V+\operatorname{dim}(1-a) V \\
& =\left(j^{2}+\left(\begin{array}{l}
j \\
3
\end{array}\right)\right) k+j^{3} k-\left(\begin{array}{l}
j \\
3
\end{array}\right) k=j^{2}(j+1) k .
\end{aligned}
$$

2. Formal power series in two variables, Akivis algebras. We consider a field $F$ of arbitrary characteristic and a vector space $W$ over $F$. A monomial function $f: W \times W \rightarrow W$ in $x$ and $y$ of degree $n=1,2, \ldots$ is a function for which there is a multilinear map $\Phi: W^{p} \times W^{q} \rightarrow W$ with $f(x, y)=\Phi((x, \ldots, x),(y, \ldots, y)), p+q=n$. We say that $f: W \times W$ $\rightarrow W$ is a homogeneous polynomial in $x$ and $y$ of degree $n$ if it is a finite linear combination of monomial functions of degree $n$. We consider constants as degree 0 polynomials. We say that a sequence $\left(f_{0}, f_{1}, \ldots\right)$ of homogeneous polynomials of $x$ and $y$ of degree $\operatorname{deg} f_{n}=n$ is a formal power series in $x$ and $y$ which we write $f=f_{0}+f_{1}+\cdots$. Clearly, formal power series in $x$ and $y$ form an $F$-vector space. (It should be clear how one defines formal power series in any finite set of variables.)

We shall consider a formal power series in $x$ and $y$ which we shall denote $x \circ y$, and for which we postulate

$$
x \circ 0=0 \circ x=x \text { for all } x .
$$

We then note

(2) $x \circ y=x+y+q(x, y)+r(x, x, y)+s(x, y, y)+f_{4}+\cdots$

with a bilinear monomial $q$ and trilinear monomials $r$ and $s$.

From $\S 1$ we recall that $f_{3}$ does not determine the pair $(r, s) \in$ $\operatorname{Hom}\left(W^{3} ; W\right)$ uniquely, but only up to pairs $(r, s)$ such that $r$ is antisymmetric in the first two variables and $s$ is antisymmetric in the second two. 
If $f=f_{1}+f_{2}+\cdots+$ is a power series with zero constant term, then substitutions of the type $x \circ f(x, y)$ and $f(x, y) \circ y$ are well defined. By induction, we construct unique power series $x / y$ and $x \backslash y$ satisfying the equations

$$
(x / y) \circ y=x \text { and } x \circ(x \backslash y)=y .
$$

(Indeed, if e.g. $x / y=p_{1}+p_{2}+\cdots$ with homogeneous polynomials $p_{n}$ which are to be determined, we obtain equations $p_{1}+y=x, p_{2}+$ $q\left(p_{1}, y\right)=0, \quad p_{3}+q\left(p_{2}, y\right)+r\left(p_{1}, p_{1}, y\right)+s\left(p_{1}, y, y\right)=0, \ldots$ which allow a unique successive solution

$$
\begin{gathered}
p_{1}=x-y, \quad p_{2}=-q(x, y)+q(y, x), \\
p_{3}=-q(q(x, y), y)+q(q(y, x), x)-r(x-y, x-y, y) \\
-s(x-y, y, y), \ldots)
\end{gathered}
$$

On account of (1), (2) and (3) we shall call the pair ( $\left.W,^{\circ}\right)$ a formal loop.

By straightforward calculation we obtain

2.1. LEMmA. The power series $(x \circ y) /(y \circ x),(y \circ x) \backslash(x \circ y)$, and $(x \circ y)-(y \circ x)$ all have vanishing degree 1 term and the same degree 2 component $q(x, y)-q(y, x)$.

The following is a bit more technical, but presents no essential difficulty:

2.2. LEMMA. The power series

$$
((x \circ y) \circ z) /(x \circ(y \circ z)),(x \circ(y \circ z)) \backslash((x \circ y) \circ z)
$$

and $(x \circ y) \circ z-x \circ(y \circ z)$ have all vanishing degree 1 and 2 components and the same degree 3 component

$$
\begin{aligned}
q(q(x, y), z)-q(x, q(y, z))+r(x, y, z) & +r(y, x, z) \\
& -s(x, y, z)-s(x, z, y) .
\end{aligned}
$$

Proof. We record the calculation for the last power series: Firstly, we calculate up to degree 3:

$$
\begin{aligned}
u= & (x \circ y) \circ z=x+y+q(x, y)+r(x, x, y)+s(x, y, y)+z \\
& +q(x+y, z)+q(q(x, y), z)+r(x+y, x+y, z)+s(x+y, z, z) \\
= & x+y+z+q(x, y)+q(x, z)+q(y, z)+q(q(x, y), z) \\
& +r(x, x, y)+r(x, x, z)+r(x, y, z)+r(y, x, z)+r(y, y, z) \\
& +s(x, y, y)+s(x, z, z)+s(y, z, z) .
\end{aligned}
$$


Secondly and similarly we calculate up to degree 3 :

$$
\begin{aligned}
v= & x \circ(y \circ z)=x+y+z+q(x, y)+q(x, z)+q(y, z) \\
& +q(x, q(y, z))+r(x, x, y)+r(x, x, z)+r(y, y, z) \\
& +s(x, y, y)+s(x, y, z)+s(x, z, y)+s(x, z, z)+s(y, z, z) .
\end{aligned}
$$

Subtracting the two polynomials gives us precisely the vanishing of the degree 1 and 2 components and the degree 3 component (4) of $u-v$, the last of the three power series in the Lemma.

If we consider the first of the three power series, then the linear term $u-v$ in the expansion of $u / v$ give us (4). The quadratic term $-q(u, v)+$ $q(v, u)$ yields vanishing components of order 2 and 3 , since the linear and quadratic terms of $u$ and $v$ agree, and the higher order components do not concern us here. In a similar vein, the third order term $f_{3}(u, v)$ of $u / v$ cannot bring any contribution since only the degree 1 components of $u$ and $v$ play a role here, and they are equal: indeed $f_{3}(w, w)$ is clearly 0 .

Through the preceding calculation we have associated with the degree 2 and 3 components of the given power series $x \circ y$ a bilinear map in Lemma 2.1 and a trilinear map in Lemma 2.2. These measure the "degree of commutativity and associativity" of the power series operation $x \circ y$ and thus deserve special attention.

2.3. Definition. With a power series $x \circ y=x+y+q(x, y)+$ $r(x, x, y)+s(x, y, y)+\cdots$ we associate (a) the commutator $[x, y]=$ $q(x, y)-q(y, x)$ and (b) the associator

$$
\begin{aligned}
\langle x, y, z\rangle= & q(q(x, y), z)-q(x, q(y, z))+r(x, y, z) \\
& +r(y, x, z)-s(x, y, z)-s(x, z, y) .
\end{aligned}
$$

Thus we have equipped the vector space $W$ with a binary and ternary operation. If, by chance, $W$ is a Lie algebra over a field of characteristic 0 and if $q(x, y)=\frac{1}{2}[x, y], r(x, y, z)=s(y, z, x)=\frac{1}{12}[x,[y, z]], f_{n}=H_{n}$ so that $x \circ y$ is the Campbell-Hausdorff series $x * y$, then we have by a well-known basic property of this series the associative identity $(x \circ y) \circ z$ $=x \circ(y \circ z)$, whence $\langle x, y, z\rangle=0$ by Lemma 2.2, and the commutator is the given Lie bracket by Lemma 2.1. What is the general situation? As a guiding principle, let us consider an $F$-algebra $E$ with a binary bilinear multiplication $(x, y) \mapsto x y: E \times E \rightarrow E$. We then define

$$
[x, y]=x y-y x \text { and }\langle x, y, z\rangle=(x y) z-x(y z) \text {, }
$$


thereby creating a new binary multiplication and a new ternary trilinear operation. We define two further ternary trilinear operations from the bracket $[x, y]$ by setting

$$
\begin{aligned}
K & =K(x, y, z)=[[x, y], z] \text { and } \\
J & =J(x, y, z)=[[x, y], z]+[[y, z], x]+[[z, x], y] .
\end{aligned}
$$

We will also abbreviate $\langle x, y, z\rangle$ by $A_{0}=A(x, y, z)$. Let $V$ again denote the $F$-vector space of all trilinear maps $E^{3} \rightarrow E$. Then $S_{3}$ acts on $V$ as in $\S 1$, paragraph (6). We can rewrite the definition of $J$ as

$$
J=\left(1+c+c^{2}\right) K \text { with the cyclic permutation } c=\left(\begin{array}{lll}
1 & 2 & 3
\end{array}\right) .
$$

We note that the element $6 a=\sum_{g \in S_{3}} \operatorname{sgn}(g) g$ is well-defined for any characteristic.

2.4. LEMMA. Let $p \in(1-a) V$ be any trilinear map $E^{3} \rightarrow E$ without non-trivial antisymmetric part and define $A=A_{0}+p$ (i.e., $A(x, y, z)=$ $\langle x, y, z\rangle+p(x, y, z))$. Then

(A) $6 a A=J$, equivalently,

$$
\sum_{g \in S_{3}} \operatorname{sgn}(g) A\left(x_{g(1)}, x_{g(2)}, x_{g(3)}\right)=J\left(x_{1}, x_{2}, x_{3}\right) .
$$

Proof. Since $p \in(1-a) V$ we have $a p=0$, so it obviously suffices to verify (A) with $A_{0}$ in place of $A$.

If we set $u(x, y, z)=(x y) z$ and $v(x, y, z)=x(y z)$, then $A_{0}=u-v$, and we note that with $t=\left(\begin{array}{ll}1 & 2\end{array}\right)$ and $c=\left(\begin{array}{lll}1 & 2 & 3\end{array}\right)$ in $S_{3}$, we have $K=$ $u-t u-(c v-c t v)$, for $u-t u=(x y) z-(y x) z$ and $c v-c t v=$ $z(x y)-z(y x)$. Then

$$
\begin{aligned}
J & =\left(1+c+c^{2}\right) K \\
& =\left(1+c+c^{2}-t-c t-c^{2} t\right)-\left(c+c^{2}+1-c t-c^{2} t-t\right) v \\
& =6 a(u-v)=6 a A_{0} .
\end{aligned}
$$

This leads us to the following Definition:

2.5. Definition. An Akivis algebra $L$ over a field $F$ of any characteristic is a $F$-vector space together with a binary bilinear antisymmetric multiplication $(x, y) \mapsto[x, y]$ and a ternary, trilinear multiplication $(x, y, z) \mapsto\langle x, y, z\rangle$ such that the identity (A) links the two operations. The relation (A) is called the Akivis identity. The Akivis algebra is called alternative iff the ternary multiplication $A=\langle x, y, z\rangle$ is antisymmetric, 
i.e., is in $a V$, where $V$ is the space of all trilinear maps $L \times L \times L \rightarrow L$. This is tantamount to saying that $a A=A$ or, in other words, that $\langle x, x, y\rangle=\langle x, y, x\rangle=\langle x, y, y\rangle=0$. We call an Akivis algebra powerassociative iff $\langle x, x, x\rangle=0$ for all $x \in L$.

We noted in Lemma 2.4 that for any binary algebra $E$ we have constructed an Akivis algebra $E_{A}=(E,[\cdot, \cdot],\langle\cdot, \cdot, \cdot\rangle)$, which we call the Akivis algebra associated with $E$.

A few simple remarks are in order:

2.6. REMARKs. 1. If $L$ is an alternative Akivis algebra over a field of char $\mathrm{F} \neq 2,3$ then $\langle x, y, z\rangle=\frac{1}{6} \mathrm{~J}(x, y, z)$. Thus the ternary operation is completely determined by the bracket operation, and the algebra may be considered as a binary algebra whose associator is defined by $\langle x, y, z\rangle$ $=\frac{1}{6} J(x, y, z)$, and which then satisfies (A).

2. If $E$ is a binary algebra which is alternative in the sense that the associativity condition $x(y z)=(x y) z$ holds whenever two of the arguments agree, then the associated Akivis algebra $\mathrm{E}$ is alternative. If $E$ is power-associative in the sense that each element generates an associative subalgebra, then $E_{A}$ is power-associative.

3. If $E$ is binary algebra with an antisymmetric multiplication $(x, y)$ $\mapsto[x, y]$, then the definition $\langle x, y, z\rangle=\frac{1}{6} J(x, y, z)$ turns $E$ into an alternative Akivis algebra $(E,[\cdot, \cdot],\langle\cdot, \cdot, \cdot\rangle)$.

Proof. 1. Since $L$ is alternative, and in view of the Akivis identity, we have $J=6 a A=A$. We leave 2 and 3 as exercises. (Cf. [12], IX.6.5.)

The gist of these observations is that in the context of alternative structures, no harm comes from forgetting the ternary multiplication, because it can be reconstituted from the binar: one. In particular, every Lie algebra is an Akivis algebra with $A=0$, and conversely, an Akivis algebra with $A=0$ is nothing but a Lie algebra.

Now we have the concepts for clarifying the link between the commutator and the associator associated with a power series (for a differential geometric version see Akivis [3], Theorem 5):

2.7. THEOREM I. Let $W$ be a vector space over the field $F$ of characteristic $\neq 2,3$ and let

$$
x \circ y=x+y+q(x, y)+r(x, x, y)+s(x, y, y)+f_{4}+\cdots
$$


be a formal power series with a bilinear map $q$ and two trilinear maps $r$ and s. If $[x, y]$ and $\langle x, y, z\rangle$ denote the commutator and associator associated with the formal loop $(W, \circ)$ according to $2.1,2.2$, and 2.3 , then writing $L(W, \circ):=(W,[\cdot, \cdot],\langle\cdot, \cdot, \cdot\rangle)$ we find that $L(W, \circ)$ is an Akivis algebra. If the power series $x \circ y$ is such that $(x \circ y) \circ z=x \circ(y \circ z)$ holds whenever two (respectively, all three) of $x, y$ and $z$ are equal, then $L(W, \circ)$ is alternative (resp., power-associative). If $(x \circ y) \circ z=x \circ(y \circ z)$ for all $x, y, z$, then $L\left(W,{ }^{\circ}\right)$ is a Lie algebra.

Proof. We let $A=\langle x, y, z\rangle$ and write $A_{q}(x, y, z)=q(q(x, y), z)-$ $q(x, q(y, z))$ for the associator bracket for the binary multiplication $q$. Then by 2.3 we have

$$
A=A_{q}+(1+t) r-(1+t c) s
$$

$$
\text { with } t=\left(\begin{array}{ll}
1 & 2
\end{array}\right), c=\left(\begin{array}{lll}
1 & 2 & 3
\end{array}\right), t c=\left(\begin{array}{ll}
2 & 3
\end{array}\right) \text { in } S_{3} \text {. }
$$

If we write $p=(1+t) r-(1+t c) s(=F(r, s)$ in the notation of $\S 1$ !) then $a p=a(1+t) r-a(1+t c) s=0$. Then Lemma 2.4 shows that $6 a A$ $=J_{q}$ where $J_{q}=\left(1+c+c^{2}\right) K_{q}$ with $K_{q}(x, y, z)=\left[[x, y]_{q}, z\right]_{q}$, where $[x, y]_{q}=q(x, y)-q(y, x)$. But by Lemma 2.1. we know $q(x, y)-$ $q(y, x)=[x, y]$, whence $J_{q}=J$. Thus the Akivis identity is proved. If $x \circ(y \circ z)=(x \circ y) \circ z$ whenever two of $x, y, z$ are equal, then by Lemma 2.2, we find that $\langle x, y, z\rangle=0$ whenever two of $x, y, z$ are equal. The proof of Theorem 1 is complete.

In Theorem 1 we associate with a formal loop $(W, \circ)$ an Akivis algebra $L(W, \circ)$. To what extent, if at all, is the converse true: Given an Akivis algebra $E$, is there a formal loop $(W, \circ)$ such that $L(W, \circ) \cong E$ ?

2.8. TheOREM II. Let $W$ be an Akivis algebra over a field $F$ with characteristic $\neq 2,3$. Then there is a polynomial in $x$ and $y$ of total degree 3 :

$$
x \circ y=x+y+\frac{1}{2}[x, y]+r(x, x, y)+s(x, y, y)
$$

such that the formal loop $(W, \circ)$ satisfies $L(W, \circ) \cong W$.

Let $U$ denote the vector space of all homogeneous polynomials $p(x, y)$ in $x$ and $y$ of degree 3 with $p(x, 0)=p(0, x)=0$, and $U_{W}$ the set of all $p \in U$ such that the definition $x \circ y=x+y+\frac{1}{2}[x, y]+p(x, y)$ implies $L(W, \circ)$ $\cong W$. Then $U_{W}$ is an affine variety in $U$ whose translation group is isomorphic to the vector space $m V$ of all symmetric trilinear maps $W^{3} \rightarrow W$. If $\operatorname{dim} W=j$ is finite, then $\operatorname{dim} m V=j^{2}\left(j^{2}-\frac{1}{6}(j-1)(j-2)\right)$. 
Proof. We let $V$ be the vector space of all trilinear maps $W^{3} \rightarrow W$. Again we define $F: V \oplus V \rightarrow V$ by $F(r, s)=(1+t) r-(1+t c) s$. According to Lemma 2.4, using the fact that $q(x, y)-q(y, x)=2 q(x, y)$ for any antisymmetric map $q$, we must find pairs $(r, s) \in V \oplus V$ such that

$$
\begin{aligned}
\langle x, y, z\rangle= & \frac{1}{2}\left[\frac{1}{2}[x, y], z\right]-\frac{1}{2}\left[x, \frac{1}{2}[y, z]\right]+r(x, y, z) \\
& +r(y, x, z)-s(x, y, z)-s(x, z, y) .
\end{aligned}
$$

This is equivalent to

$$
\begin{aligned}
& A=\frac{1}{4}(1+c) K+F(r, s) ; \quad \text { equivalently, } \\
& F(r, s)=A-\frac{1}{4}(1+c) K
\end{aligned}
$$

The Akivis identity says $6 a A=J$. We always have $6 a(1+c) K=12 a K$ $=2\left(\left(1+c+c^{2}\right) K-\left(1+c+c^{2}\right) t K\right)=4 J$, i.e. $3 a(1+c) K=2 J$. Thus the Akivis identity is equivalent to $6 a\left(A-\frac{1}{4}(1+c) K\right)=$ $J-\frac{2}{3} a(1+c) K=0$, i.e. to

$$
A-\frac{1}{4}(1+c) K \in(1-a) V .
$$

But by the results of $\S 1$, notably 1.3 and 1.4 , we know that $(1-a) V=$ $\operatorname{im} F$, whence the desired existence of $(r, s)$ follows.

Now let $p \in U$ be any polynomial of degree 3 such that $x \circ y=$ $x+y+\frac{1}{2}[x, y]+p(x, y)$ gives us $L(W, \circ) \cong W$. If $p^{\prime}$ is another one with the same property, then $p-p^{\prime}$ is in the kernel of the map $\phi: U \rightarrow$ $(1-a) V$ of 1.6. By 1.6, this kernel is isomorphic to $m V$, the space of all symmetric trilinear maps $W^{3} \rightarrow W$. In the finite dimensional case, the dimension of this space was given at the end of $\S 1$.

It is precisely in the proof of this Theorem that we use the background information which we prepared in $\$ 1$. We note that Theorem II is indeed a converse of Theorem $I$, but it lacks a precise uniqueness statement. The existence of the desired power series $x \circ y$ can be secured even in the realm of polynomials of degree at most 3 , but even in this domain there is no uniqueness; the ambiguity is of the magnitude of the space of all symmetric trilinear maps of $W^{3}$ into $W$.

We notice that the power series $x / y$ and $y \backslash x$ constructed for $x \circ y$ as in (3) above will not be polynomial even though $x \circ y$ is a polynomial of total degree 3 . In view of the fact, that for a Lie algebra $W$ there is one and only one power series $x * y$ with $(x * y) * z=x *(y * z)$ such that $L(W, *) \cong W$, namely, the Campbell-Hausdorff series, it is certainly 
desirable to have more information on the relation between Theorems I and II above and these classical results. Our understanding of the situation is limited at this time. However, the following remarks are certainly pertinent.

Firstly, the Campbell-Hausdorff series is inseparably linked with the exponential function. Formally, the handling of the exponential function requires the ability to deal with formal power series on $W$ of the form $a_{0}+a_{1} x+a_{2} x^{2}+\cdots+a_{n} x^{n}+\cdots$ with $a_{n} \in F$. Here it is an indispensable hypothesis that we can talk about powers $x^{n}$. This means that we are operating in an algebra $W$ with a binary multiplication $(x, y) \rightarrow x y$ which is at least power-associative (see [25], p. 128). In a power-associative algebra $W$ we can now indeed define a power series

$$
\begin{aligned}
x * y=H_{1}(x, y)+H_{2}(x, y)+\cdots & =L((\exp x \exp y)-1), \\
\text { where } L(z) & =z-\frac{1}{2} z^{2}+\frac{1}{3} z^{3}-+\cdots,
\end{aligned}
$$

and where the $H_{n}(x, y)$ are homogeneous polynomials in $x$ and $y$ of degree $n$ which are linear combinations of monomials in $x$ and $y$ of degree $n$, i.e., words in the free binary algebra generated by two letters. Thus $H_{1}(x, y)=x+y$ and $H_{2}(x, y)=\frac{1}{2}(x y-y x)$ are verified without undue pain. The calculation of $H_{3}(x, y)$ is already painful, but instructive. In fact we have

2.9. Proposition (Holmes and Sagle [13], Theorem 3.6). Let $W$ be a power-associative algebra over a field $F$ of characteristic 0 and let $[x, y]=$ $x y-y x$ and $\langle x, y, z\rangle=(x y) z-x(y z)$ be the operations in the associated Akivis algebra $W_{A}$. Then the homogeneous component of degree three in the Campbell-Hausdorff series has the form

$$
H_{3}(x, y)=\frac{1}{12}([x,[x, y]]+[y,[y, x]])+P_{3}(x, y)
$$

with

$$
\begin{aligned}
P_{3}(x, y) & =\frac{2}{3}\left(\langle x, x, y\rangle-\langle x, y, y\rangle-\frac{1}{3}(\langle y, y, x\rangle-\langle y, x, x\rangle)\right) \\
& =\frac{1}{3}(\langle x, x, y\rangle-\langle x, y, x\rangle-\langle x, y, y\rangle+\langle y, x, y\rangle) .
\end{aligned}
$$

This Proposition was first proved by Holmes and Sagle in the context of calculus in $n$-dimensional real vector spaces. Our proof is merely a question of book-keeping. The recipe is as follows: We set

$$
\begin{aligned}
z & =(\exp x \exp y)-1 \\
& =x+y+\frac{1}{2} x^{2}+x y+\frac{1}{2} y^{2}+\frac{1}{6} x^{3}+\frac{1}{2} x y^{2}+\frac{1}{2} x^{2} y+\frac{1}{6} y^{3}
\end{aligned}
$$


up to terms of higher order. We then have to consider $x * y=z-\frac{1}{2} z^{2}$ $+\frac{1}{3} z^{3}$ up to terms of higher order. This forces us to calculate the homogeneous components of degree 3 in the power series $z^{2}$ and $z^{3}$ which leads to the following table:

\begin{tabular}{|c|c|c|c|c|c|c|c|c|c|c|c|c|}
\hline$(z)_{3}$ & $\begin{array}{l}x^{3} \\
=\overline{\frac{1}{6}}=\end{array}$ & $\begin{array}{l}y^{3} \\
=\overline{\frac{1}{6}}=\end{array}$ & $\begin{array}{l}x y^{2} \\
=\frac{1}{2}=\end{array}$ & $\begin{array}{l}x^{2} y \\
=\overline{\frac{1}{2}}=\end{array}$ & $y^{2} x$ & $\begin{array}{l}y x^{2} \\
==\end{array}$ & $\begin{array}{l}(x y) y \\
===\end{array}$ & $\begin{array}{l}(x y) x \\
==\end{array}$ & $\begin{array}{l}y(x y) \\
==\end{array}$ & $\begin{array}{l}x(x y) \\
===\end{array}$ & $\begin{array}{l}x(y x) \\
===\end{array}$ & $\begin{array}{l}y(y x) \\
===\end{array}$ \\
\hline$\left(z^{2}\right)_{3}$ & 1 & 1 & $\frac{1}{2}$ & $\frac{1}{2}$ & $\frac{1}{2}$ & $\frac{1}{2}$ & 1 & 1 & 1 & 1 & & \\
\hline$\left(z^{3}\right)_{3}$ & 1 & 1 & 1 & & & 1 & & & 1 & 1 & 1 & 1 \\
\hline
\end{tabular}

We must now calculate $(z)_{3}-\frac{1}{2}\left(z^{2}\right)_{3}+\frac{1}{3}\left(z^{3}\right)_{3}$. The terms $x^{3}$ and $y^{3}$ drop out. There is no canonical way to proceed from here: however we suggest to do the accounting as follows: We calculate the polynomial in question as a linear combination of $x y^{2}, x(x y), x(y x), y x^{2}, y(y x)$, $y(x y),\langle x, x, y\rangle=x^{2} y-(x y) y,\langle x, y, y\rangle=(x y) y-x y^{2},\langle x, y, x\rangle=$ $(x y) x-x(y x)$, and $\langle y, y, x\rangle=y^{2} x-y(y x)$. This calls for some rewriting of $(z)_{3}$ and $\left(z^{2}\right)_{3}$, but not of $\left(z^{3}\right)_{3}$. Now we collect the coefficients in the following table:

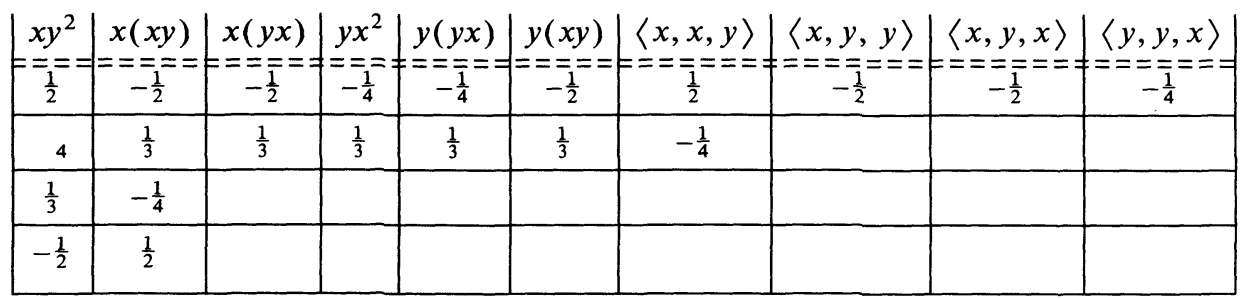

Now we have the following information on the series $x * y$ :

$$
\begin{aligned}
x * y= & x+y+\frac{1}{2}[x, y]+\frac{1}{12} x y^{2}+\frac{1}{12} x(x y)-\frac{1}{6} x(y x) \\
& +\frac{1}{12} y x^{2}+\frac{1}{12} y(y x)-\frac{1}{6} y(x y)+\frac{1}{4}\langle x, x, y\rangle-\frac{1}{2}\langle x, y, y\rangle \\
& -\frac{1}{2}\langle x, y, x\rangle-\frac{1}{4}\langle y, y, x\rangle+\text { terms of higher order } \\
= & x+y+\frac{1}{2}[x, y]+\frac{1}{12}\left(x y^{2}+x(x y)+y x^{2}+y(y x)\right. \\
& \quad-x(y x)-x(y x)-y(x y)-y(x y)) \\
& +\frac{3}{4}\langle x, x, y\rangle-\frac{1}{2}\langle x, y, y\rangle+\frac{1}{2}\langle y, x, x\rangle-\frac{1}{4}\langle y, y, x\rangle \\
& + \text { terms of higher order, }
\end{aligned}
$$

since

$$
\langle x, x, y\rangle+\langle y, x, x\rangle+\langle x, y, x\rangle=0
$$


as a consequence of power-associativity, which yields $\langle x \pm y, x \pm y$, $x \pm y\rangle=0$. We now eliminate the remaining monomials $x y^{2}$ etc. by introducing brackets in a suitable fashion. We note e.g. that

$$
\begin{aligned}
x(x y)-x & (y x)-x(y x)+y x^{2} \\
& =x[x, y]-(x y) x+(y x) x+\langle x, y, x\rangle-\langle y, x, x\rangle \\
& =x[x, y]-[x, y] x+\langle x, y, x\rangle-\langle y, x, x\rangle \\
& =[x,[x, y]]+\langle x, y, x\rangle-\langle y, x, x\rangle \\
& =[x,[x, y]]-(\langle x, x, y\rangle+2\langle y, x, x\rangle)
\end{aligned}
$$

(again with the aid of (14)). In a similar fashion, we calculate

$$
\begin{aligned}
x y^{2}+y(y x)-y(x y)-y(x y) & =[y,[y, x]]-\langle x, y, y\rangle+\langle y, x, y\rangle \\
& =[y,[y, x]]-(\langle y, y, x\rangle+2\langle x, y, y\rangle) .
\end{aligned}
$$

If we substitute these expressions into (13) we obtain the asserted form of $H_{3}(x, y)$.

We notice that $P_{3}(x, y)=0$, whenever $W$ is an associative algebra, and then $H_{3}(x, y)$ becomes the usual homogeneous component of degree 3 in the classical Campbell-Hausdorff formula.

The preceding proposition suggests that for a given Akivis algebra $E$ we consider that polynomial

$$
x \circ y=x+y+\frac{1}{2}[x, y]+r(x, x, y)+s(x, y, y)
$$

for which the $r$ and $s$ are chosen so that

$$
H_{3}(x, y)=r(x, x, y)+s(x, y, y), \quad \text { with } H_{3}(x, y) \text { as in 2.9. }
$$

If we set

$$
\begin{aligned}
& r(x, y, z)=\frac{1}{12}[x,[y, z]]+\frac{2}{3}\langle x, y, z\rangle+\frac{1}{3}\langle z, x, y\rangle, \text { and } \\
& s(x, y, z)=\frac{1}{12}[y,[z, x]]-\frac{2}{3}\langle x, y, z\rangle-\frac{1}{3}\langle y, z, x\rangle,
\end{aligned}
$$

then we verify that (16) implies (15). Now the associated Akivis algebra $L(E, \circ)$ has the same commutator as $E$, but the associator is different. A straightforward but somewhat lengthy calculation based on (9) yields

$$
A_{0}=\langle x, y, z\rangle_{0}=\frac{1}{2} J+\left(1+t+c t+c^{2} t\right) A \text { with } A=\langle x, y, z\rangle .
$$

Equivalently, we can express the deviation of the given Akivis algebra $E$ from the Akivis algebra $L(E, \circ)$ by the difference

$$
A_{0}-A=\left(1+c+c^{2}\right) A-\frac{1}{2} J \text {. }
$$


We recall $e=\frac{1}{3}\left(1+c+c^{2}\right)$ from $\S 1$ and then note that $L(E, \circ)=E$ iff

(AA) $e A=\frac{1}{6} J$, equivalently,

$$
\begin{aligned}
& \langle x, y, z\rangle+\langle y, z, x\rangle+\langle z, x, y\rangle \\
& \quad=\frac{1}{2}([[x, y], z]+[[y, z], x]+[[z, x], y]),
\end{aligned}
$$

which is "half of the Akivis identity (A)". Of course, (AA) implies (A), but not inversely as is readily verified in the Akivis algebra associated with the free binary algebra in three generators $x, y$, and $z$. Thus, in the absence of condition (AA), the Campbell-Hausdorff formula does not yield a power series for which $L(E, *)=E$.

If $E$ is alternative, then $A=\frac{1}{6} J$ by 2.6.1. Then $e A=\frac{1}{6} e J=\frac{1}{6} J$ and thus (AA) is satisfied.

More generally, if $E$ is an alternative Akivis algebra, then the Akivis subalgebra generated by two elements $x$ and $y$ is a Lie algebra. Hence the homogeneous polynomials $H_{n}(x, y)$ of the Campbell-Hausdorff series are simply the ones known from Lie algebra theory. This gives us the following remark:

2.10. Proposition. If $W$ is an alternative Akivis algebra over a field of characteristic 0 , then the classical Campbell-Hausdorff series $x * y$ yields a formal loop $(W, *)$ with the property that $L(W, *)=W$. The same holds for any power series $x \circ y$ which agrees with $x * y$ up to homogeneous components of degree 1,2 and 3.

Several pressing questions remain open.

2.11. Problems. [12] 1. Let $W$ be the free power-associative algebra in two indeterminates $x$ and $y$ over a field of characteristic 0 . In the associated Akivis algebra $W_{A}$ we denote by $\operatorname{Ak}[x, y]$ the Akivis subalgebra generated by $x$ and $y$. If $H_{n}(x, y)$ are the polynomials determined as the homogeneous components of the formal power series $x * y=$ $L((\exp x \exp y)-1)$, is it true that

$$
H_{n}(x, y) \in \operatorname{Ak}[x, y],
$$

i.e. that the $H_{n}(x, y)$ are linear combinations of monomials built up from commutator and associator brackets alone? (The answer is yes for $n=$ 1, 2, 3 by 2.9.)

2. Is $\operatorname{Ak}[X]$, the Akivis subalgebra generated by the set $X$ of free generators of a free algebra $W$ inside the associated Akivis algebra $W_{A}$ the free Akivis algebra over $X$ ? 
3. Let $\operatorname{fr}(W)$ denote the free non-associative algebra over a vector space $W$. If $W$ carries the structure of an Akivis algebra, we denote with $I$ the ideal generated in $\operatorname{fr}(W)$ by the set of all $x y-y x-[x, y]$ and $(x y) z-x(y z)-\langle x, y, z\rangle$ with $x, y, z \in W$ and define $U(W)=\operatorname{fr}(W) / I$ and let $f_{W}: W \rightarrow U(W)$ be defined by $f_{W}(w)=w+I$. Then for any (not necessarily associative) algebra $E$ and any morphism of Akivis algebras $h$ : $W \rightarrow E_{A}$ there is a unique morphism $h^{\prime}: U(W) \rightarrow E$ such that $h^{\prime} f_{W}=h$. We call $U(W)$ the enveloping algebra of the Akivis algebra $W$. Under which circumstances if $f$ injective? Is there a parallel to the theorem of Poincaré, Birkhoff and Witt (cf. [9], §§2, 7) in the context of enveloping algebras of Akivis algebras? Is $\operatorname{fr}(X)=U(A k[X])$ ? (Cf. also [3], Problem 1.)

3. Lie's fundamental theorems for local analytical loops. In this section we let $F$ denote the real field $\mathbf{R}$ or the complex field $\mathbf{C}$. (We are aware of the fact that other fields such as e.g. $\mathbf{Q}_{p}$ could be included, too.) Let $W$ be a completely normable $F$-vector space. A monomial function $f$ : $W \times W \rightarrow W$ of degree $n=1,2,3, \ldots$ is a function for which there is a continuous multilinear map $\Phi: W^{p} \times W^{q} \rightarrow W$ with $f(x, y)=$ $\Phi((x, \ldots, x),(y, \ldots, y)), p+q=n$. We say that $f: W \times W \rightarrow W$ is a homogeneous polynomial function of degree $n$ if it is a finite linear combination of monomial functions of degree $n$. We say that a function $f: B \times B \rightarrow W$ is analytic (with an open neighbourhood $B$ of 0 in $W$ ) whenever there is a formal power series $f_{0}+f_{1}+f_{2}+\cdots$ whose homogeneous components $f_{n}$ are homogeneous polynomial functions such that the infinite series $f_{0}+f_{1}(x, y)+f_{2}(x, y)+\cdots$ converges absolutely on $B \times B$ relative to a norm on $W$ compatible with the topology.

3.1. Definition. A local analytical loop is a completely normable real or complex vector space $W$ together with an open neighbourhood $B$ of 0 and with three analytical function $\circ, /, \backslash: B \times B \rightarrow W$ such that the following identities are satisfied:

(i) $x \circ 0=0 \circ x=x$ for all $x \in B$

(ii) $x \circ(x \backslash y)=(y / x) \circ x=y$ for all $x, y \in B$ with $x \backslash y, y / x \in B$.

It follows from Definition 3.1 and the early observation (2) of $\S 2$, that the local multiplication of a local analytical loop is given by

$x \circ y=x+y+q(x, y)+r(x, x, y)+s(x, y, y)+f_{4}(x, y)+\cdots$ for $(x, y) \in B \times B$ with continuous multilinear maps $q: W^{2} \rightarrow W$ and $r, s: W^{3} \rightarrow W$. 
3.2. Proposition. Let $W$ be a completely normable F-vector space and

$$
\begin{aligned}
(x, y) \mapsto & x \circ y=x+y+q(x, y)+r(x, x, y)+s(x, y, y) \\
& +f_{4}(x, y)+\cdots: B_{0} \times B_{0} \rightarrow W
\end{aligned}
$$

an analytical function with multilinear continuous maps $q, r, s$ and an open neighbourhood of 0 in $W$, so that $x \circ 0=0 \circ x$ for all $x$ sufficiently close to 0 . Then there is an open neighbourhood $B$ of 0 in $B_{0}$ and analytical functions $/, \backslash: B \times B \rightarrow W$ such that $\left(W, B,{ }_{B}, /, \backslash\right)$ is a local analytical loop with the restriction ${ }^{\circ} B=\circ \mid(B \times B)$.

Proof. We consider the function $m: B_{0} \times B_{0} \rightarrow B_{0} \times W$, given by $m(x, y)=(x, x \circ y)$. This function is analytic and has the derivative $\operatorname{Dm}(0)$ given by $\operatorname{Dm}(0)(x, y)=(x, x+y)$ which is an isomorphism of vector spaces. Hence $m$ allows a local analytical inverse by the inverse function theorem (see e.g. [8], 5.7.6.) We note that $m^{-1}(x, y)=(x, u)$ means $(x, y)=m(x, u)=(x, x \circ u)$; thus if we write $u=x \backslash y$, then the analytical function $(x, y) \mapsto x \backslash y$ satisfies the equation $x \circ(x \backslash y)=y$ on its domain. In a similar fashion one obtains $x / y$. If we choose $B$ small enough so that all functions in question are defined on $B \times B$, we have completed the proof.

3.3. Proposition. Let $(W, B, \circ, /, \backslash)$ be a local analytical loop. Then the following limits all exist and satisfy the identities

$$
\begin{gathered}
\lim _{t \rightarrow 0} t^{-2}((t x \circ t y) /(t y \circ t x))=\lim _{t \rightarrow 0} t^{-2}((t y \circ t x) \backslash(t x \circ t y)) \\
=\lim _{t \rightarrow 0} t^{-2}(t x \circ t y-t y \circ t x)=q(x, y)-q(y, x)
\end{gathered}
$$

$$
\begin{aligned}
& \lim _{t \rightarrow 0} t^{-3}(((t x \circ t y) \circ t z) /(t x \circ(t y \circ t z))) \\
&= \lim _{t \rightarrow 0} t^{-3}((t x \circ(t y \circ t z)) \backslash((t x \circ t y) \circ t z)) \\
&= \lim _{t \rightarrow 0} t^{-3}((t x \circ t y) \circ t z-t x \circ(t y \circ t z)) \\
&= q(q(x, y), z)-q(x, q(y, z))+r(x, y, z) \\
&+r(y, x, z)-s(x, y, z)-s(x, z, y) .
\end{aligned}
$$

Proof. This follows from the definitions in 3.1, the subsequent observation (1), and Lemmas 2.1 and 2.2.

3.4. Definition. Under the circumstances of Proposition 3.3 we call the bilinear expression in relation (3) the commutator bracket of the loop 
and denote it by $[x, y]$, and we call the trilinear expression in relation (4) the associator bracket of the loop and denote it by $\langle x, y, z\rangle$.

3.5. Definition. A topological Akivis algebra is a topological $F$-vector space $W$ together with two continuous multilinear maps $[\cdot, \cdot]: W^{2} \rightarrow W$ and $\langle\cdot, \cdot, \cdot\rangle: W^{3} \rightarrow W$ which satisfy the Akivis identity.

3.6. Proposition. Let $(W, B, \circ, /, \backslash)$ be a local analytical loop. Then the commutator bracket and associator bracket give continuous multilinear maps $[\cdot, \cdot]: W^{2} \rightarrow W$ and $\langle\cdot, \cdot, \cdot\rangle: W^{3} \rightarrow W$, respectively, and $(W,[\cdot, \cdot]$, $\langle\cdot, \cdot, \cdot\rangle)$ is a topological Akivis algebra.

Proof. From the fact that $q, r$ and $s$ are continuous multilinear maps and from Propositin 3.3 it is immediate that the commutator and associator brackets are continuous multilinear maps. From Theorem 2.7 we know that $(W,[\cdot, \cdot],\langle\cdot, \cdot, \cdot\rangle)$ is an Akivis algebra whenever we have the relations $[x, y]=q(x, y)-q(y, x)$ and

$$
\begin{aligned}
\langle x, y, z\rangle= & q(q(x, y), z)-q(x, q(y, z))+r(x, y, z) \\
& +r(y, x, z)-s(x, y, z)-s(x, z, y)
\end{aligned}
$$

with multilinear functions $q, r$, and $s$.

If $(W, B, \circ, /, \backslash)$ is a local analytical loop, then we say that the topological Akivis algebra $(W,[\cdot, \cdot],\langle\cdot, \cdot, \cdot\rangle)$ is the Akivis algebra associated with the loop and write $L(W, B, \circ, /, \backslash)=(W,[\cdot, \cdot],\langle\cdot, \cdot, \cdot\rangle)$. If no confusion arises we will briefly write $L(B, \circ)$.

We are now ready for the fundamental theorems.

\subsection{LIE'S FUNDAMENTAL THEOREMS FOR LOCAL ANALYTICAL LOOPS.}

A. Every local analytical loop $(W, B, \circ, /, \backslash)$ determines a unique associated topological Akivis algebra $L(B, \circ)=(W,[\cdot, \cdot],\langle\cdot, \cdot, \cdot\rangle)$ (whose commutator and associator brackets are given by (3) and (4) above).

B. Let $W=(W,[\cdot, \cdot],\langle\cdot, \cdot, \cdot\rangle)$ be a topological Akivis algebra on a completely normable real or complex vector space. Then there is a local analytical loop $(W, B, \circ, /, \backslash)$ such that $L(B, \circ)=W$. Moreover, there exist such loops for which the multiplication $\circ$ is polynomial of the form (5) $x \circ y=x+y+\frac{1}{2}[x, y]+r(x, x, y)+s(x, y, y)$ with continuous multilinear maps $r$, s. The set of differences $x \circ_{2} y-x \circ_{1} y$ of such multiplications is in bijective correspondence with the vector space of all symmetric continuous trilinear maps $W^{3} \rightarrow W$. 
Proof. Part A is proved by Proposition 3.3. Part B follows from Theorem 2.8 which gives us two continuous trilinear maps $r$ and $s$ such that, with $q(x, y)=\frac{1}{2}[x, y]$ for $x \circ y$ given by (5), we have $\langle x, y, z\rangle$ equal to the expression in equation (4). Proposition 2.3 gives us the analytical local quotient functions / and \, which will make $(W, B, \circ, /, \backslash)$ a local analytical loop. The remainder now follows from Theorem 2.8 .

3.8. Definition. A local analytical loop $(W, B, \circ, /, \backslash)$ is said to be $a$ local analytical group if (6) $(x \circ y) \circ z=x \circ(y \circ z)$ holds whenever all products in this formula are defined. A subset $C \subseteq B$ is called a local subloop iff $(C \circ C) \cap B \subseteq C,(C / C) \cap B \subseteq C$ and $(C \backslash C) \cap B \subseteq C$. Also $C$ is called a local subgroup if it is a local subloop and (6) holds whenever $x, y, z \in C$ and all products in the formula are defined.

We say that $(W, B, \circ, /, \backslash)$ is an alternative (power-associative) local analytical loop if every set $X \subseteq B$ of at most two elements (respectively, one element) lies in a local subgroup.

3.9. Proposition. A. The Akivis algebra of a local analytical alternative loop is alternative. B. If $W$ is an alternative topological Akivis algebra on a completely normable vector space, then there is a local analytical alternative loop $(W, B, *, /, \backslash)$ where $x * y=x+y+\frac{1}{2}[x, y]+H_{3}(x, y)$ $+\cdots$ is given by the classical Campbell-Hausdorff series converging absolutely on $B$ (which we may take an open ball of radius $\frac{1}{2} \log 2$; here we single out a norm compatible with the topology and which satisfies $\|[x, y]\| \leq$ $\|x\|\|y\|)$ and where $x / y=y \backslash x=x-y$, and $L(B, *)=W$.

Proof. A. Proposition 3.3 shows immediately that $\langle x, y, z\rangle$ vanishes whenever $\{x, y, z\}$ contains at most two elements. B. follows from Proposition 2.10 and the classical circle of ideas around the CampbellHausdorff formula.

There is a considerable theory on Moufang Lie loops and the corresponding Akivis algebras which are known as Malcev algebras in the literature [12 through 16, 19 through 23]. Moufang Lie loops are alternative and Malcev algebras are alternative (here the expression binary Lie algebra is also used in the literature instead of "alternative Akivis algebra").

We recall that in the classical local Lie group theory the Lie algebra determines a local Lie group up to local isomorphism. This fact was first extended to alternative Lie loops by Malcev [20]. Through the work of 
Holmes and Sagle (see [13]) one knows that a power-associative local analytical loop may always be parametrized in such a fashion that it takes the form $(\mathrm{W}, \mathrm{B}, \circ, /, \backslash)$ with $x \circ y=x+y$, whenever $x$ and $y$ are linearly dependent. This then holds, in particular, for alternative local analytical loops. If $(\mathrm{W}, \mathrm{B}, \circ, /, \backslash)$ is an alternative local analytical loop with $x \circ y=x+y$ for linearly dependent $x, y \in W$ then $x \circ y$ must agree with the Campbell-Hausdorff product $x * y$ for all sufficiently small $x, y$. For if $x$ and $y$ are two elements in $B$ and if we assume that $u * v$ is defined for all $u, v \in B$, then there is a closed vector subspace $V$ of $W$ containing $x$ and $y$ on which the Akivis algebra structure $L(W, \circ)$ of $W$ induces the structure of a Lie algebra, and for which $\circ$ induces on $V \cap B$ the structure of a local analytical group with $x \circ y=x+y$ for linearly dependent $x$ and $y$. Classical Lie group theory then yields $u \circ v=u * v$ on $V$ for sufficiently small $u$ and $v$. Since $\circ$ and $*$ are both analytic, they agree on their common domain. Since $x$ and $y$ were arbitrary in $B$, the claim is established.

There are other applications of the canonical coordinates introduced by Holmes and Sagle for power-associative local analytical loops.

3.10. RemarK. (Cf. [12], IX.6.23). If ( $W, B, \circ, /, \backslash)$ is a power-associative local analytical loop satisfying the identity $x^{-1}(x y)=(y x) x^{-1}=$ $y$, then $W$ is alternative.

It is no problem to carry out the theory outlined in this section for local loops of class $C^{k}, k=3,4, \ldots$, instead of class $C^{\omega}$ which we discussed here.

\section{REFERENCES}

[1] M. A. Akivis, Canonical expansions of the equations of a local analytic quasi-group, (Russian). Dokl. Akad. Nauk SSSR, 188 (1969), 967-970. Translated in: Sov. Math., 10 (1969), 1200-1203.

[2] Local differentiable quasigroups and three-webs of multidimensional surfaces, (Russian). In: Studies in the Theory of Quasigroups and Loops, (Russian), Stiintsa: Kishinev, (1973), 3-12.

[3] _ Local algebras of a multidimensional web, Sib. Mat. Zh., 17 (1976), 5-11. Translated in Sib. Math. J., 17 (1976), 3-8.

[4] M. A. Akivis and A. M. Shelekhov, The calculation of curvature and torsion tensor for a multidimensional 3-web and the associator of its local quasigroup, Sib. Mat. Zh., 12 (1971), 953-960. Translated in Sib. Math. J., 12 (1972), 685-689.

[5] On local differentiable quasigroups and connections related to a 3-web of multidimensional surfaces, Sib. Mat. Zh., 12 (1971), 1181-1191. Translated in Sib. Math. J., 12 (1972), 845-852.

[6] Local analytic quasigroups and loops, (Russian), Kalinin Gosud-Univ., Kalinin, (1980), 31 pp. 

, Foundation of the web theory, (Russian), Kalinin Gosud. Univ., Kalinin, (1981), $88 \mathrm{pp}$.

[8] N. Bourbaki, Variétés différentielles et analytiques, Fasc. des résultats. Éléments de mathématiques 33. Hermann: Paris 1967.

[9] __ Groupes et Algèbres de Lie: Chapitre 1. Éléments de mathématique 26. Hermann: Paris 1960.

[10] Groupes et Algèbres de Lie: Chapitres 2 et 3, Éléments de mathématique 37, Hermann: Paris 1972.

[11] K. H. Hofmann and J. D. Lawson, On Sophus Lie's Fundamental Theorems, I, Indag. Math., 45 (1983), 453-466.

[12] K. H. Hofmann and K. Strambach, Topological and analytical quasigroups and loops and related structures. In: The Theory of Quasigroups and Loops, Chapter IX, Heldermann Verlag, Berlin, to appear.

[13] J. P. Holmes and A. A. Sagle, Analytic $H$-spaces, Campbell-Hausdorff formula, and alternative algebras, Pacific J. Math., 91 (1980), 105-134.

[14] F. S. Kerdman, Analytic Moufang loops in the large, Algebra i Logika, 18 (5) (1979), 523-555. Translated in: Algebra and Logic, 18 (1980), 325-347.

[15] E. N. Kuzmin, Mal'cev algebras and their representations, Algebra i Logika, 7 (4) (1968), 48-69. Translated in: Algebra and Logic, 7 (1968), 233-254.

[16] _ The connection between Mal'cev algebras and analytic Moufang loops, (Russian), Algebra i Logika, 10 (1971), 3-22. Translated in: Algebra and Logic, 10 (1972), 1-14.

[17] _ Levi's theorem for Mal'cev algebras, (Russian), Algebra i Logika, 16 (4) (1977), 424-431. Translated in: Algebra and Logic, 16, (1978), 286-291.

[18] S. Lie, Vorlesungen über kontinuierliche Gruppen mit geometrischen und anderen Anwendungen, Leipzig 1893. Reprint Chelsea Publishing Company: New York 1971.

[19] S. MacLane, Homology. Die Grundlehren der Mathematischen Wissenschaften in Einzeldarstellungen. Bd. 114. Springer: Berlin-Göttingen-Heidelberg 1963.

[20] A. I. Mal'cev, Analytical loops, Math. Sb., 36 (1955), 569-576.

[21] L. V. Sabinin and P. O. Mikheev, Analytic Bol loops, (Russian). In: Webs and quasigroups, pp. 102-109. Kalinin Gos. Univ., Kalinin 1982.

[22] A. A. Sagle, Mal'cev algebras, Trans. Amer. Math. Soc., 101 (1961), 426-458.

[23] _ Simple Mal'cev algebras over fields of characteristic zero, Pacific J. Math., 12 (1962), 1057-1078.

[24] , On anti-commutative algebras and analytic loops, Canad. J. Math., 17 (1965), $550-558$.

[25] R. D. Schafer, An Introduction to Nonassociative Algebras, Academic Press: New York and London 1966.

[26] J.-P. Serre, Lie Algebras and Lie Groups, Lectures Given at Harvard University. Benjamin: New York-Amsterdam 1965.

Received August 16, 1984 and in revised form October 9, 1984.

TeChNische Hochschule Darmstadt

SCHLOßGARTENSTRAßE 7

D-6100 Darmstadt, Germany, FRG

AND

UNIVERSITÄT ERLANGEN-NUURNBERG

BISMARCKSTR. $1 \frac{1}{2}$

D-8520 ERLANGEN, GERMANY, FRG 



\title{
PACIFIC JOURNAL OF MATHEMATICS EDITORS
}

\author{
V. S. VARADARAJAN \\ (Managing Editor) \\ University of California \\ Los Angeles, CA 90024 \\ HERBERT ClEMENS \\ University of Utah \\ Salt Lake City, UT 84112 \\ R. FINN \\ Stanford University \\ Stanford, CA 94305
}

\author{
HERMANN FLASCHKA \\ University of Arizona \\ Tucson, AZ 85721 \\ RAMESH A. GANGOLLI \\ University of Washington \\ Seattle, WA 98195 \\ VAUGHAN F. R. JONES \\ University of California \\ Berkeley, CA 94720 \\ ROBION KIRBY \\ University of California \\ Berkeley, CA 94720
}

C. C. MOORE

University of California

Berkeley, CA 94720

H. SAMELSON

Stanford University

Stanford, CA 94305

HAROLD STARK

University of California, San Diego

La Jolla, CA 92093

\section{ASSOCIATE EDITORS}

\author{
R. AREnS \\ E. F. BECKENBACH \\ B. H. NEUMANN \\ F. WOLF \\ K. YOSHIDA \\ (1906-1982)

\section{SUPPORTING INSTITUTIONS}

\begin{abstract}
UNIVERSITY OF ARIZONA
UNIVERSITY OF BRITISH COLUMBIA

UNIVERSITY OF CALIFORNIA

MONTANA STATE UNIVERSITY

UNIVERSITY OF NEVADA, RENO

NEW MEXICO STATE UNIVERSITY

OREGON STATE UNIVERSITY
\end{abstract} \\ CALIFORNIA INSTITUTE OF TECHNOLOGY \\ UNIVERSITY OF OREGON \\ UNIVERSITY OF SOUTHERN CALIFORNIA \\ STANFORD UNIVERSITY \\ UNIVERSITY OF HAWAII \\ UNIVERSITY OF TOKYO \\ UNIVERSITY OF UTAH \\ WASHINGTON STATE UNIVERSITY \\ UNIVERSITY OF WASHINGTON
}

The Supporting Institutions listed above contribute to the cost of publication of this Journal, but they are not owners or publishers and have no responsibility for its content or policies.

Mathematical papers intended for publication in the Pacific Journal of Mathematics should be in typed form or offset-reproduced (not dittoed), double spaced with large margins. Please do not use built up fractions in the text of the manuscript. However, you may use them in the displayed equations. Underline Greek letters in red, German in green, and script in blue. The first paragraph must be capable of being used separately as a synopsis of the entire paper. In particular it should contain no bibliographic references. Please propose a heading for the odd numbered pages of less than 35 characters. Manuscripts, in triplicate, may be sent to any one of the editors. Please classify according to the scheme of Math. Reviews, Index to Vol. 39. Supply name and address of author to whom proofs should be sent. All other communications should be addressed to the managing editor, or Elaine Barth, University of California, Los Angeles, California 90024.

There are page-charges associated with articles appearing in the Pacific Journal of Mathematics. These charges are expected to be paid by the author's University, Government Agency or Company. If the author or authors do not have access to such Institutional support these charges are waived. Single authors will receive 50 free reprints; joint authors will receive a total of 100 free reprints. Additional copies may be obtained at cost in multiples of 50 .

The Pacific Journal of Mathematics is issued monthly as of January 1966. Regular subscription rate: $\$ 190.00$ a year (5 Vols., 10 issues). Special rate: $\$ 95.00$ a year to individual members of supporting institutions.

Subscriptions, orders for numbers issued in the last three calendar years, and changes of address should be sent to Pacific Journal of Mathematics, P.O. Box 969, Carmel Valley, CA 93924, U.S.A. Old back numbers obtainable from Kraus Periodicals Co., Route 100, Millwood, NY 10546.

The Pacific Journal of Mathematics at P.O. Box 969, Carmel Valley, CA 93924 (ISSN 0030-8730) publishes 5 volumes per year. Application to mail at Second-class postage rates is pending at Carmel Valley, California, and additional mailing offices. Postmaster: send address changes to Pacific Journal of Mathematics, P.O. Box 969, Carmel Valley, CA 93924.

PUBLISHED BY PACIFIC JOURNAL OF MATHEMATICS, A NON-PROFIT CORPORATION

Copyright (C) 1986 by Pacific Journal of Mathematics 


\section{Pacific Journal of Mathematics}

\section{Vol. 123, No. $2 \quad$ April, 1986}

David Jay Anick, A loop space whose homology has torsion of all orders . . 257 Steven P. Diaz, Space curves that intersect often ................. 263

Thierry Fack and Hideki Kosaki, Generalized $s$-numbers of $\tau$-measurable

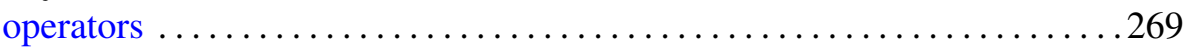

Karl Heinrich Hofmann and Karl Strambach, Lie's fundamental

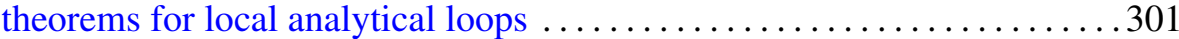

James Secord Howland, On the Kato-Rosenblum theorem ............. 329

Frieder Knüppel and Edzard Salow, Plane elliptic geometry over rings . . . . 337

Alan Noell, Peak points in boundaries not of finite type ................ 385

William J. Ralph, An extension of singular homology to Banach

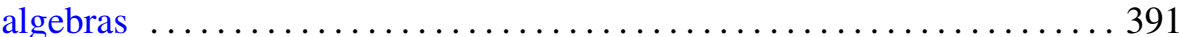

Wade C. Ramey, Averaging properties of pluriharmonic boundary values . . 407

Thomas Joseph Ransford, On the range of an analytic multivalued

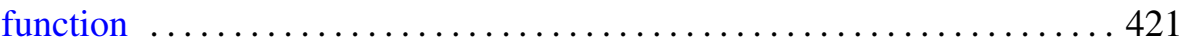

Christopher Donald Sogge, On restriction theorems of maximal-type . . . . 441

Edwin Spanier, Cohomology with supports $\ldots \ldots \ldots \ldots \ldots \ldots \ldots \ldots \ldots 47$

Emil J. Straube, Orthogonal projections onto subspaces of the harmonic

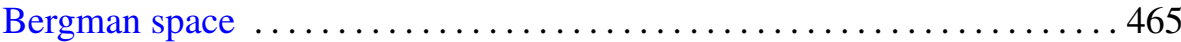

Thomas Vogel, Asymptotic behavior of two semilinear elliptic free boundary problems $\ldots \ldots \ldots \ldots \ldots \ldots \ldots \ldots \ldots$ 